\title{
Salidroside induces apoptosis and autophagy in human colorectal cancer cells through inhibition of PI3K/Akt/mTOR pathway
}

\author{
XIANG-JUN FAN, YAO WANG, LEI WANG and MINGYAN ZHU \\ Department of General Surgery, Affiliated Hospital of Nantong University, Nantong, Jiangsu 226001, P.R. China
}

Received May 15, 2016; Accepted September 16, 2016

DOI: $10.3892 /$ or. 2016.5138

\begin{abstract}
The role of salidroside in colon cancer remains unknown. Here we show that salidroside, a phenylpropanoid glycoside extracted from Rhodiola rosea, exhibited potent anti-proliferative properties in human colorectal cancer cells via inducing apoptosis and autophagy. We ascertained that salidroside exerts an inhibitory effect on the proliferation of human colorectal cancer cells in a dose-dependent manner. In addition, salidroside induced cell apoptosis, accompanied by an increase of chromatin condensation and nuclear fragmentation, and a decrease of $\mathrm{Bcl}-2 / \mathrm{Bax}$ protein expression ratio. We also found that salidroside induced autophagy, evidenced by increased $\mathrm{LC}^{+}$autophagic vacuoles, positive acridine orange-stained cells, enhanced conversion of LC3-I to LC3-II, and elevation of Beclin-1. Treatment with autophagy-specific inhibitors [3-methyladenine (3-MA) and bafilomycin A1 (BA)] enhanced salidroside-induced apoptosis, indicating that salidroside-mediated autophagy may protect HT29 cells from undergoing apoptotic cell death. Additionally, salidroside decreased the phosphorylation of PI3K, Akt and mTOR. Treatment with PI3K inhibitor LY294002 augmented the effects of salidroside on the expression of Akt and mTOR. These findings indicate that salidroside could suppress the $\mathrm{PI} 3 \mathrm{~K} / \mathrm{Akt} / \mathrm{mTOR}$ signaling pathways. This study may provide a rationale for future clinical application using salidroside as a chemotherapeutic agent for human colorectal cancer.
\end{abstract}

\section{Introduction}

Colorectal cancer (CRC) is the most common digestive malignant and devastating primary tumor. Based on global estimates, it is the third most commonly diagnosed cancer in males and the second in females (1). Despite early diagnosis and treatment such as surgery and chemotherapy, colon cancer

Correspondence to: Dr Mingyan Zhu, Department of General Surgery, Affiliated Hospital of Nantong University, 20 Xisi Road, Nantong, Jiangsu 226001, P.R. China

E-mail: zmyntfy@163.com

Key words: autophagy, apoptosis, human colon cancer HT-29 cells, salidroside, PI3K/Akt/mTOR pathways can reappear at a later time, even if the cancer was entirely removed during the initial treatment. Therefore, the current challenge is to identify new effective less toxic chemotherapeutic agents that are need in treatment of colon cancer.

Targeting programmed cell death (PCD) has become a promising approach in the fight against cancer, which mainly includes modulation of apoptosis and autophagy $(2,3)$. Type I PCD, apoptosis, is a biological process with a crucial role in normal development and tissue homeostasis (4). Type II PCD, autophagic cell death, is a highly conserved cellular degradation process characterized by the presence of abundant intracellular autophagic vacuoles termed autophagosomes. Autophagosomes participate in the recycling of cellular components by sequestering damaged organelles and misfolded proteins, targeting them for lysosomal degradation (5-7). Apoptosis and autophagy are two distinct processes, coordinately regulating cell survival and cell death, and occur simultaneously in cancers $(8,9)$. Accumulated evidence has shown that apoptosis and autophagy is a response to various anticancer therapies in many kinds of cancer cells (10-12). In addition, the apoptosis and autophagy mechanisms are involved in CRC and play an important role in the multifactorial etiology of CRC (13). So the modulation of apoptosis and autophagy might be applied in a potential cancer therapy for the treatment of colon cancer cells.

Rhodiola rosea L, also known as 'golden root', is a perennial herbaceous plant of the Crassulaceae family, widely distributed at high-altitudes regions (14). It has long been used as adaptogen traditional Chinese medicine (15). Reports on the anticancer effect of Rhodiola extracts have been published $(16,17)$. Salidroside, a major component of Rhodiola rosea, has been reported to have significant antitumor effects, such as inhibiting cell proliferation, arresting cell cycle, and promoting apoptosis of the human bladder, breast, lung or liver cancer cells $(16,18-21)$. The existing evidence indicates that salidroside plays antitumor role by inhibiting tumor metastasis, reducing new angiogenesis and changing the tumor microenvironment (22-24). In addition, it is also reported that it can inhibit proliferation, decrease the migration and invasion of colon carcinoma SW1116 cells in JAK2/STAT3-dependent pathway (25). However, the relative molecular mechanisms still need to be studied.

Studies have found that salidroside could decrease the growth of bladder cancer cell lines via inhibition of the mTOR pathway and induction of autophagy (16). In addition, 
mTOR has emerged as an effective target for colorectal cancer therapy (26). Increasing evidence demonstrates that PI3K/Akt/ mTOR signaling plays a key role in regulation of apoptosis and autophagy, and targeting PI3K/Akt/mTOR signaling has been proposed to be a promising strategy for cancer treatment (27-29). Thus, this study aimed to investigate whether salidroside modulates apoptosis and autophagy in HT29 human colon cancer cells and to further elucidate the role of the PI3K/Akt/ mTOR signaling in regulation of cell death.

\section{Materials and methods}

Materials. Salidroside (purity $>99 \%$ ) was purchased from National Institute for the Control of Pharmaceutical and Biological Products (Beijing, China). RPMI-1640 was purchased from Gibco (Grand Island, NY, USA). Fetal bovine serum (FBS) was purchased from Sijiqing (Hangzhou, China). MTT (3-(4,5-dimethylthiazol-2-yl)-2,5-diphenyltetrazolium bromide, trypsin, Acridine orange (AO), LY294002, 3-methyladenine, Bafilomycin A1, Hoechst 33342, antibodies for the detection of LC3 (\#L7543) and Beclin-1 (\#B6186) was purchased from Sigma (St. Louis, MO, USA). Bcl-2 (\#15071), Bax (\#2772), PI3K (\#4292), p-PI3K at Tyr458 (\#4228), Akt (\#9272), p-Akt at Ser473 (\#9271), mTOR (\#2972), p-mTOR at Ser2448 (\#2971) and GAPDH (\#5174) were purchased from Cell Signaling Technology (Beverly, MA, USA). HRP-labeled goat anti-rat $\operatorname{IgG}(\mathrm{H}+\mathrm{L})$ (\#A0192), HRP-labeled goat antirabbit $\mathrm{IgG}(\mathrm{H}+\mathrm{L})$ (\#A0208), FITC-labeled goat anti-rabbit IgG (H+L) (\#A0562) were purchased from Beyotime Institution of Biotechnology (Haimen, China).

Cell culture. The human colon cancer HT-29 cells were purchased from the American Type Culture Collection (ATCC; Manassas, VA, USA). HT-29 cells were cultured in RPMI-1640 containing $10 \%$ heat-inactivated fetal bovine serum, $100 \mathrm{U} / \mathrm{ml}$ penicillin, $100 \mathrm{mg} / \mathrm{ml}$ streptomycin, and were kept at $37^{\circ} \mathrm{C}$ in a humidified atmosphere composed of $5 \% \mathrm{CO}_{2}$ and $95 \%$ air. Salidroside was diluted in cell culture medium and regulated to final concentrations of $0.5,1$ and $2 \mathrm{mM}$, and cultured for the indicated time periods. To investigate the mechanisms for salidroside-induced apoptosis and autophagy, cells were pretreated with the $10 \mu \mathrm{M}$ LY294002 (a PI3K inhibitor), $10 \mathrm{nM}$ BA (an autophagy-lysosomal inhibitor) or $10 \mathrm{mM}$ of 3-MA (an autophagy inhibitor) for $30 \mathrm{~min}$, then co-treated with $2 \mathrm{mM}$ salidroside for further $48 \mathrm{~h}$. Cells were treated with fresh medium as vehicle control.

Cell proliferation assay. Cell proliferation was assayed by MTT. Briefly, the cells were seeded in 96-well plates at a density of $1 \times 10^{4}$ cells/well. After treatment, $0.5 \mathrm{mg} / \mathrm{ml} \mathrm{MTT}$ was added to each well and the plates were incubated for another $4 \mathrm{~h}$ at $37^{\circ} \mathrm{C}$. The formazan crystals were dissolved in dimethyl sulfoxide (DMSO). Absorbance was determined at $550 \mathrm{~nm}$ on an ElX-800 MicroElisa reader (Bio-Tek Inc., Winooski, VT, USA). The cell viability were expressed as a percentage of the controls.

Hoechst 33342 staining. To quantify and assess nuclear morphology, HT29 cells were cultured on 24-well culture plates. After treatment, cells were fixed for $20 \mathrm{~min}$ with
4\% paraformaldehyde in PBS at room temperature. After staining for $10 \mathrm{~min}$ with $10 \mu \mathrm{g} / \mathrm{ml}$ Hoechst 33342, the cells were visualized and photographed under a DMR fluorescence microscope (Leica Microsystems, Wetzlar, Germany) with fluorescence excitation at $340 \mathrm{~nm}$ and emission at $510 \mathrm{~nm}$. The apoptotic index was calculated as: [apoptotic cells number / total cells number] x $100(\%)$. At least four different fields from each well were selected to count $\geq 500$ cells to calculate the rate of apoptosis.

Immunofluorescence analysis of LC3 distribution. Cells $\left(1 \times 10^{5}\right.$ cells $/ \mathrm{cm}^{2}$ in 24 -well plates) were fixed in $4 \%$ paraformaldehyde for $30 \mathrm{~min}$ at room temperature. Subsequently, the cells were permeabilized with $0.5 \%$ Triton X-100 and blocked with $1 \%$ bovine serum albumin in PBS for $1 \mathrm{~h}$, followed by incubation in anti-LC3 antibody (1:100) overnight at $4^{\circ} \mathrm{C}$, washed and incubated with FITC-labeled goat anti-rabbit IgG $(\mathrm{H}+\mathrm{L})(1: 500)$ for $2 \mathrm{~h}$ at $37^{\circ} \mathrm{C}$, rinsed with PBS, and counterstained with Hoechst 33342 for $10 \mathrm{~min}$. Images were obtained using a fluorescence microscope (488-nm filter; Olympus BX51, Japan).

Acridine orange staining. Acridine orange staining was used to detect autophagy induction. After seeding, HT-29 cells were washed with phosphate-buffered saline (PBS), stained with $1 \mu \mathrm{g} / \mathrm{ml}$ acridine orange for $15 \mathrm{~min}$ at $37^{\circ} \mathrm{C}$. Photographs were obtained with a fluorescence microscope (Axioscop, Carl Zeiss, Thomwood, NY, USA) equipped with a mercury 100-W lamp, 490-nm band-pass blue excitation filters, a 500-nm dichroic mirror and a 515-nm long-pass barrier filter. Autophagic lysosomes appeared as orange/red fluorescent cytoplasmic vesicles according to their acidity, while the nuclei were stained green.

Western blot assay. Proteins (35 $\mu \mathrm{g} / \mathrm{sample})$ were separated by SDS-PAGE and transferred onto nitrocellulose membranes (Millipore, Bedford, MA, USA). Membranes were blocked with $5 \%$ non-fat milk for $1 \mathrm{~h}$ and incubated with the following antibodies: LC3, Beclin-1, Bcl-2, Bax, PI3K, p-PI3K at Tyr458, Akt, p-Akt at Ser473, mTOR, p-mTOR at Ser2448 and GAPDH at 1:1,000 overnight at $4^{\circ} \mathrm{C}$. The membranes were washed with TBS/T (TBS with $0.05 \%$ Tween-20) and then incubated with HRP-labeled goat anti-rat $\operatorname{IgG}(\mathrm{H}+\mathrm{L})(1: 3,000)$ or HRP-labeled goat anti-rabbit $\operatorname{IgG}(\mathrm{H}+\mathrm{L})(1: 1,000)$ at room temperature for $1 \mathrm{~h}$. The reaction was visualized using ECL and detected using a Luminescent Image Analyzer LAS-4000 mini. The images were quantified with Multi Gauge. For each sample, band intensities were normalized to GAPDH.

Statistical analysis. Statistical differences were evaluated by GraphPad Prism 5.0 (San Diego, CA, USA). Statistical significance was determined by one-way analysis of variance (ANOVA) and subsequent Tukey's test. Differences were considered significant at $\mathrm{p}<0.05$. The data are expressed as mean \pm SD of three independent experiments.

\section{Results}

Salidroside inhibits growth and induces apoptosis in HT-29 colon cancer cells. HT-29 colon cancer cells were treated in indicated time periods with various concentrations of 
A
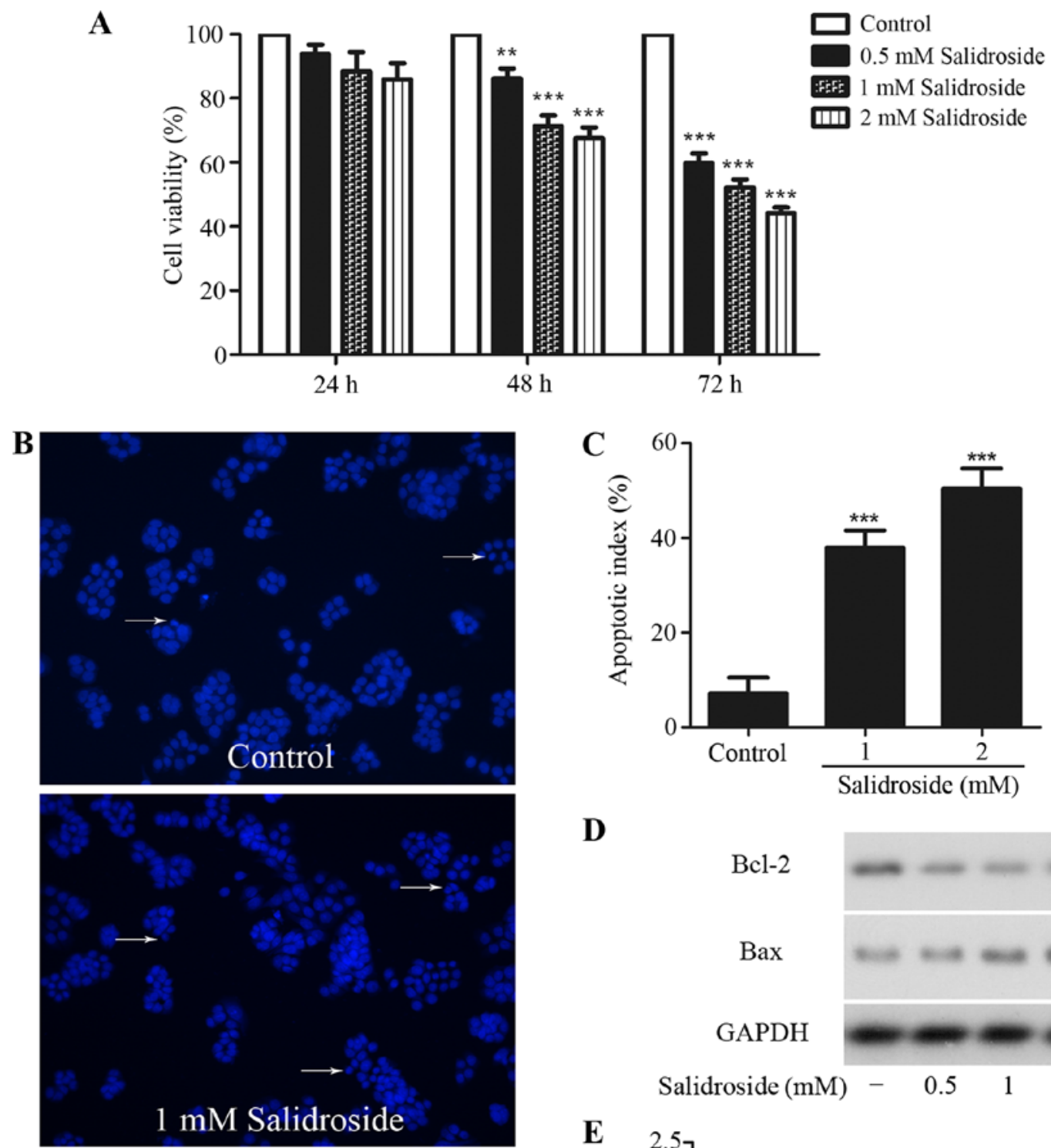

D
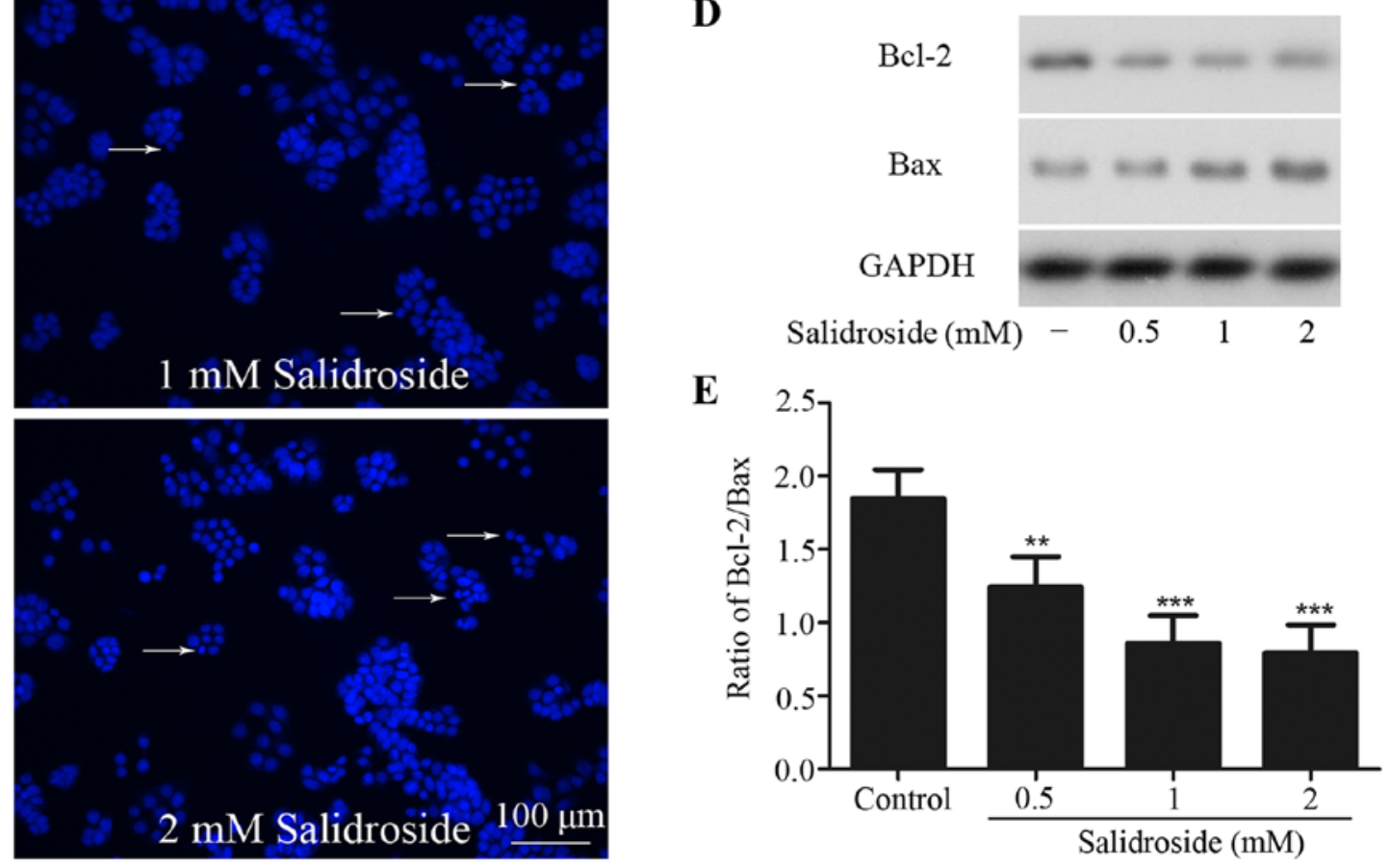

Figure 1. The ability of salidroside to induce apoptosis in HT29 colon cancer cells. (A) The HT29 cells were treated with different concentration of salidroside and incubated for different times. Cell viabilities were determined by MTT assay. (B) Hoechst 33342 staining was used to detect the morphological features of apoptosis in HT29 cells (condensed chromatin and fragmented nuclei). Arrowheads indicte apoptotic cells. (C) The apoptosis index was calculated. (D) The expression levels of Bcl-2 and Bax were analyzed by western blotting. Anti-GAPDH antibody was used for normalization. (E) The intensity of bands was quantified by densitometric analysis. All data are expressed as mean $\pm \mathrm{SD}$ from three independent experiments. ${ }^{* *} \mathrm{p}<0.01$ and ${ }^{* * * *} \mathrm{p}<0.001$ vs. control group.

salidroside to investigate the cytotoxic activity of salidroside against these cells. Cell viability was then assessed using MTT assay. MTT assay revealed a dose-dependent and timedependent cytotoxic of salidroside on these cells (Fig. 1A). Compared with control group, treatment with salidroside
$(0.5,1$ and $2 \mathrm{mM})$ for $48 \mathrm{~h}$ significantly inhibited cell viability to $86.17 \pm 6.28,71.38 \pm 6.48$ and $67.65 \pm 6.39 \%$, respectively, and $0.5,1$ and $2 \mathrm{mM}$ salidroside treatment for $72 \mathrm{~h}$ inhibited cell viability to $59.81 \pm 5.94,52.23 \pm 4.86$ and $44.12 \pm 3.71 \%$, respectively. However, treatment with salidroside for $24 \mathrm{~h}$ had 


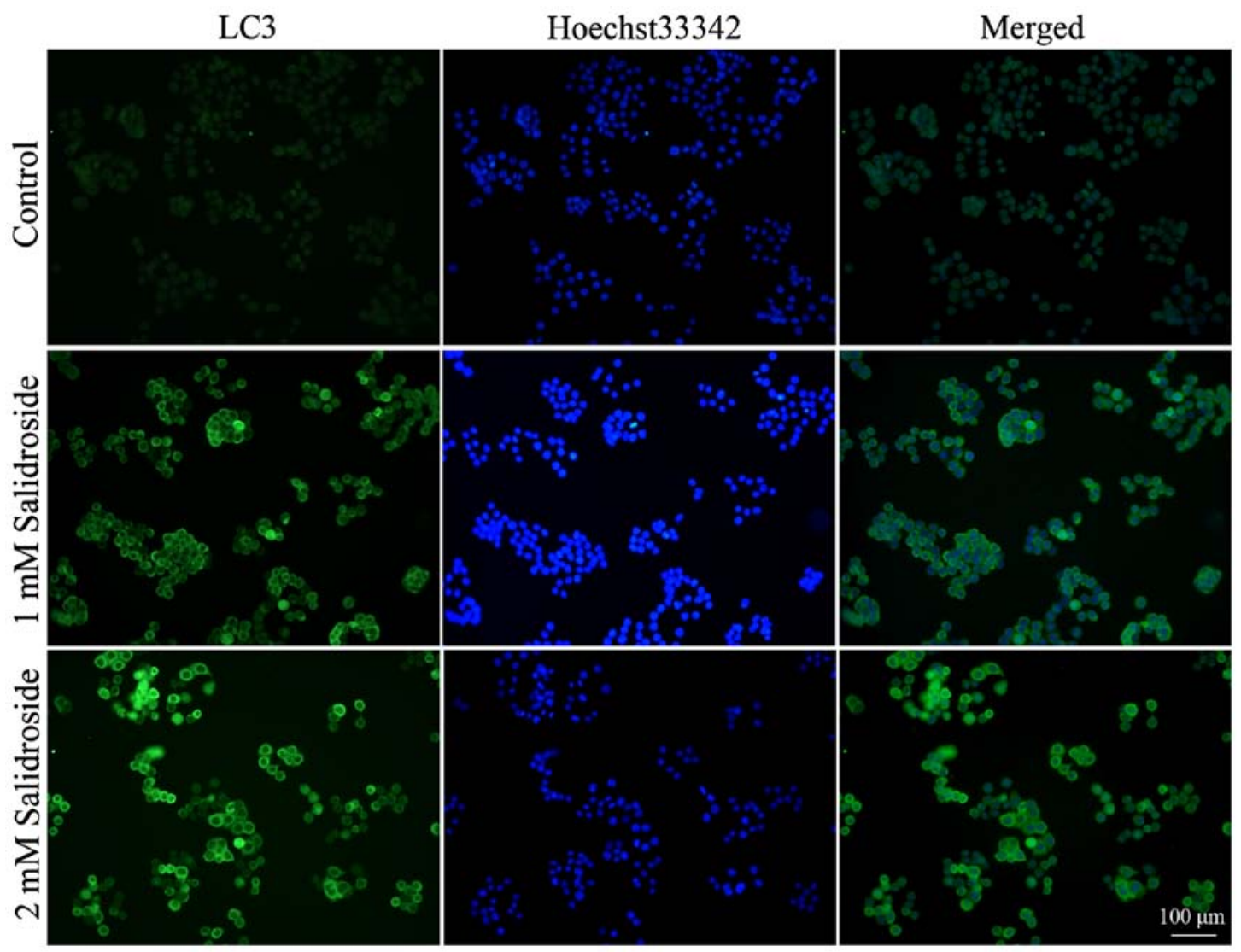

Figure 2. Salidroside induces the formation of autophagic vacuoles. Treating the cells with salidroside for $48 \mathrm{~h}$ prominently enhanced the formation of autophagic vacuoles as determined by immunofluorescent staining for LC3.

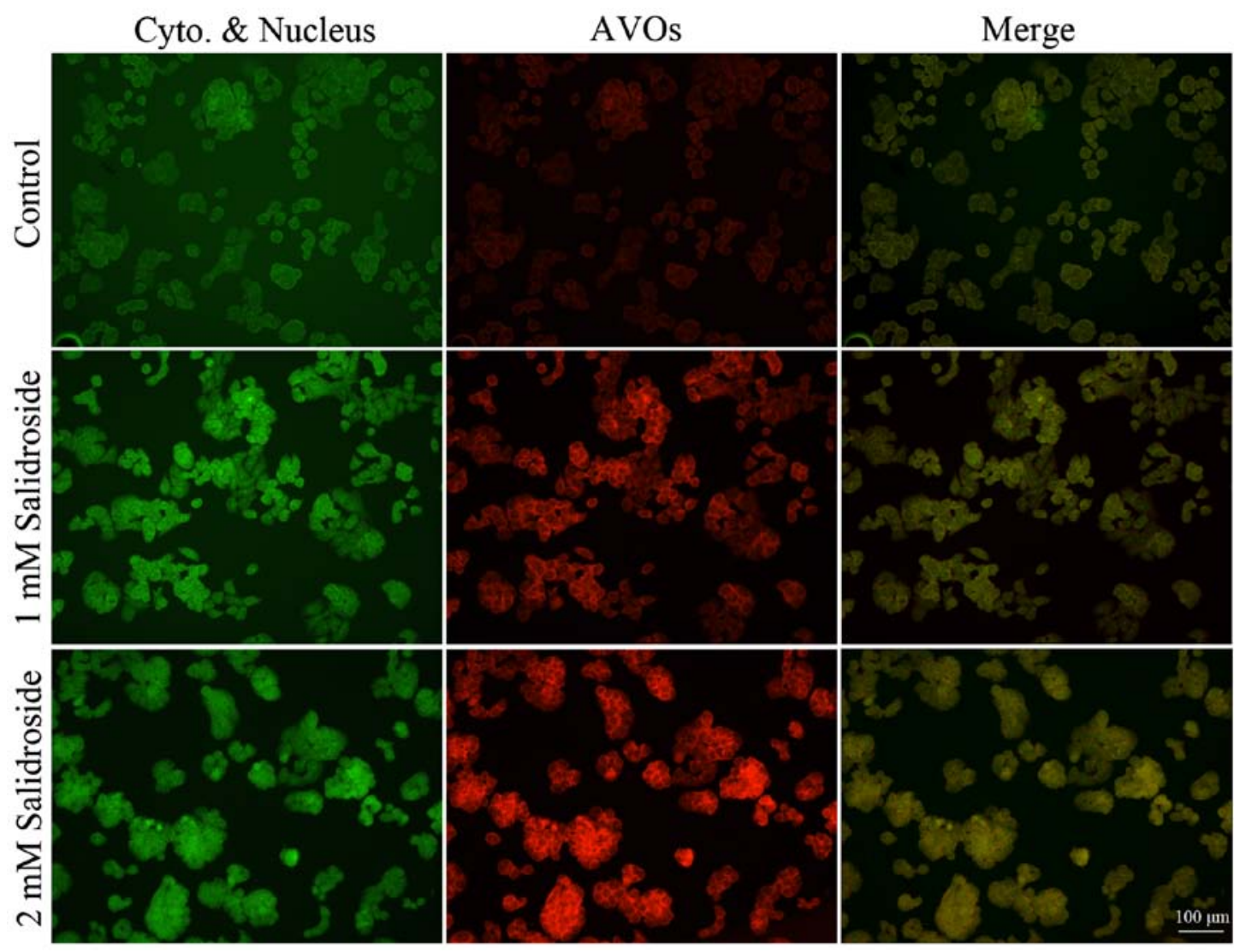

Figure 3. Salidroside induces the accumulation of acidic vesicular organelles. Cells were treated with 1 and $2 \mathrm{mM}$ salidroside for $48 \mathrm{~h}$ and stained with acridine orange. Green and red fluorescence in acridine orange-stained cells were observed under a fluorescence microscope. 
A

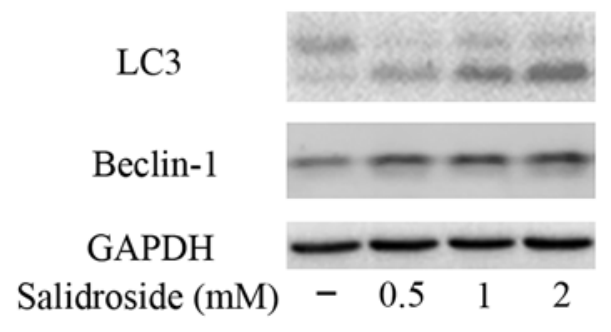

B

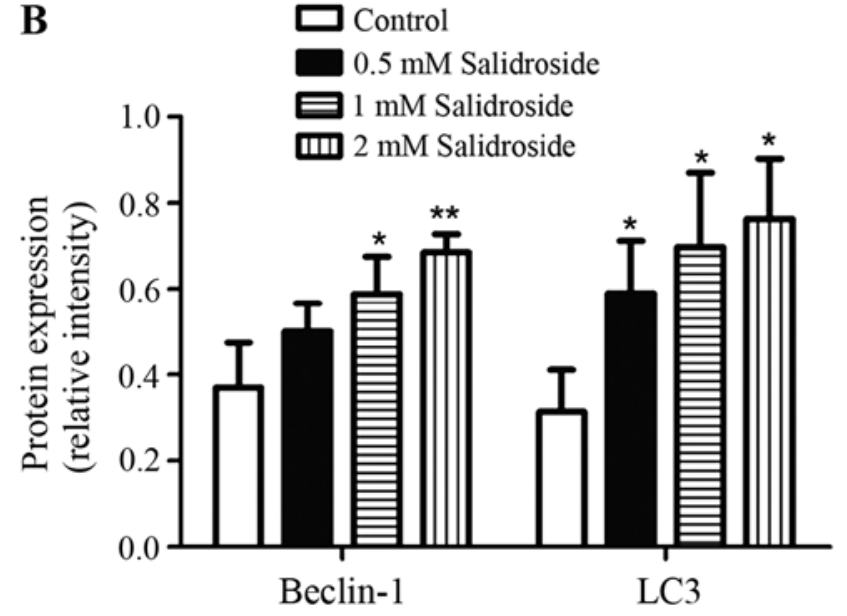

Figure 4. Effects of salidroside on the protein levels of LC3 and Beclin-1 in HT29 cells. (A) The expression levels of LC3 and Beclin-1 were analyzed by western blotting. Anti-GAPDH antibody was used for normalization. (B) The intensity of bands was quantified by densitometric analysis. All values represent mean \pm SD of three independent experiments. ${ }^{*} \mathrm{p}<0.05,{ }^{* *} \mathrm{p}<0.01$ vs. control.

no inhibitory effect. In subsequent experiments, 1 and $2 \mathrm{mM}$ salidroside treatment for $48 \mathrm{~h}$ was used to observe the effects of salidroside on HT-29 colon cancer cells.

The nuclear Hoechst 33342 staining assay was used to detect apoptosis of HT29 cells. Pretreatment with 1 and $2 \mathrm{mM}$ salidroside for $48 \mathrm{~h}$ displayed typical morphological features of apoptosis including chromatin condensation, nuclear shrinkage, and the formation of a few apoptotic bodies, and the percentage of nuclear condensation increased to $37.9 \pm 3.7$ and $50.4 \pm 4.2 \%$ from $7.1 \pm 3.4 \%$, compared with the control group (Fig. 1B and C).

Western blot analysis was used to evaluate the expression of Bcl-2 and Bax. A decreased ratio of Bcl-2/Bax was found after salidroside treatment (Fig. 1D and E). These results revealed that salidroside could induce apoptosis in HT-29 colon cancer cells.

Salidroside induces autophagy in HT-29 colon cancer cells. To determine whether salidroside induces autophagy in colon cancer cells, we used immunofluorescence to examine the intracellular distribution of LC3, an autophagy marker (30). Results showed that control HT29 colon cancer cells exhibited weak and diffuse cytoplasmic staining with LC3-associated green fluorescence, whereas those treated with salidroside exhibited an increase in LC3 staining intensity, which is a typical feature of LC3 distribution within autophagosomes (LC3-II) (Fig. 2).

To further determine the effect of salidroside on autophagy, we analyzed the accumulation of acidic vesicular organelles. Vital staining of HT-29 colon cancer cells with acridine orange revealed the appearance of acidic vesicular organelles with bright red fluorescence after salidroside treatment (Fig. 3). Conversely, the majority of control cells exhibited only minimal red fluorescence.

To clarify the mechanisms underlying the salidrosideinduced autophagy on colon cancer cells, we examined the effect of salidroside treatment on LC3 and Beclin-1 expression. It is well known that LC3-II/-I ratio directly correlates with the formation of autophagosomes (31). Lysates of cells were subjected to western blot analysis. As shown in Fig. 4, the ratio of LC3-II to LC3-I was increased by treatment with salidroside $(0.5,1$ and $2 \mathrm{mM})$ for $48 \mathrm{~h}$ compared with control. Salidroside also increased the expression of Beclin-1 compared with control.

Inhibition of autophagy enhances salidroside-induced apoptosis. As described above, we found that salidroside exhibited increased apoptosis and autophagy in HT29 cells. Then we used 3-MA (an inhibitor of autophagy) and BA (an autophagy-lysosomal inhibitor) to determine the interrelationship between apoptosis and autophagy after treating HT29 cells with salidroside. HT29 colon cancer cells treated with salidroside $(2 \mathrm{mM})$ combine with 3 -MA $(10 \mathrm{mM})$ or BA $(10 \mathrm{nM})$ decreased the formation of $\mathrm{LC}^{+}$autophagic vacuoles compared with salidroside alone (Fig. 5A). In addition, the increase of LC3-II/-I ratio and Beclin-1 protein expression by salidroside were considerably decreased by pre-treatment with 3-MA or BA, suggesting that 3-MA and BA blocked autophagy induction by salidroside. We also found that treating HT29 cells with salidroside decreased the radio of Bcl-2/Bax, which was augmented when salidroside was combined with 3-MA or BA (Fig. 5B and C). MTT assays revealed that treatment of HT29 cells with salidroside and 3-MA or BA decreased the cell viability more than treatment with salidroside alone (Fig. 5D). These results indicated that suppression of autophagy could enhance the salidroside-induced apoptosis.

Salidroside inhibits the activation of PI3K/Akt/mTOR signaling pathway. Since we have observed that salidroside could induce apoptosis and autophagy in HT29 colon cancer cells, we further investigated the possible mechanisms. The $\mathrm{PI} 3 \mathrm{~K} / \mathrm{Akt} / \mathrm{mTOR}$ signaling pathway is a key pathway related to cell survival/death $(9,32)$, then we investigated if this pathway plays a central role in salidroside-mediated cell death. As shown in Fig. 6A and B, salidroside treatment causes significant decrease in the phosphorylation levels of PI3K, Akt, and mTOR, and there was no change observed in the total PI3K, Akt, and mTOR protein level. There was a 16.9, 33.2 
A

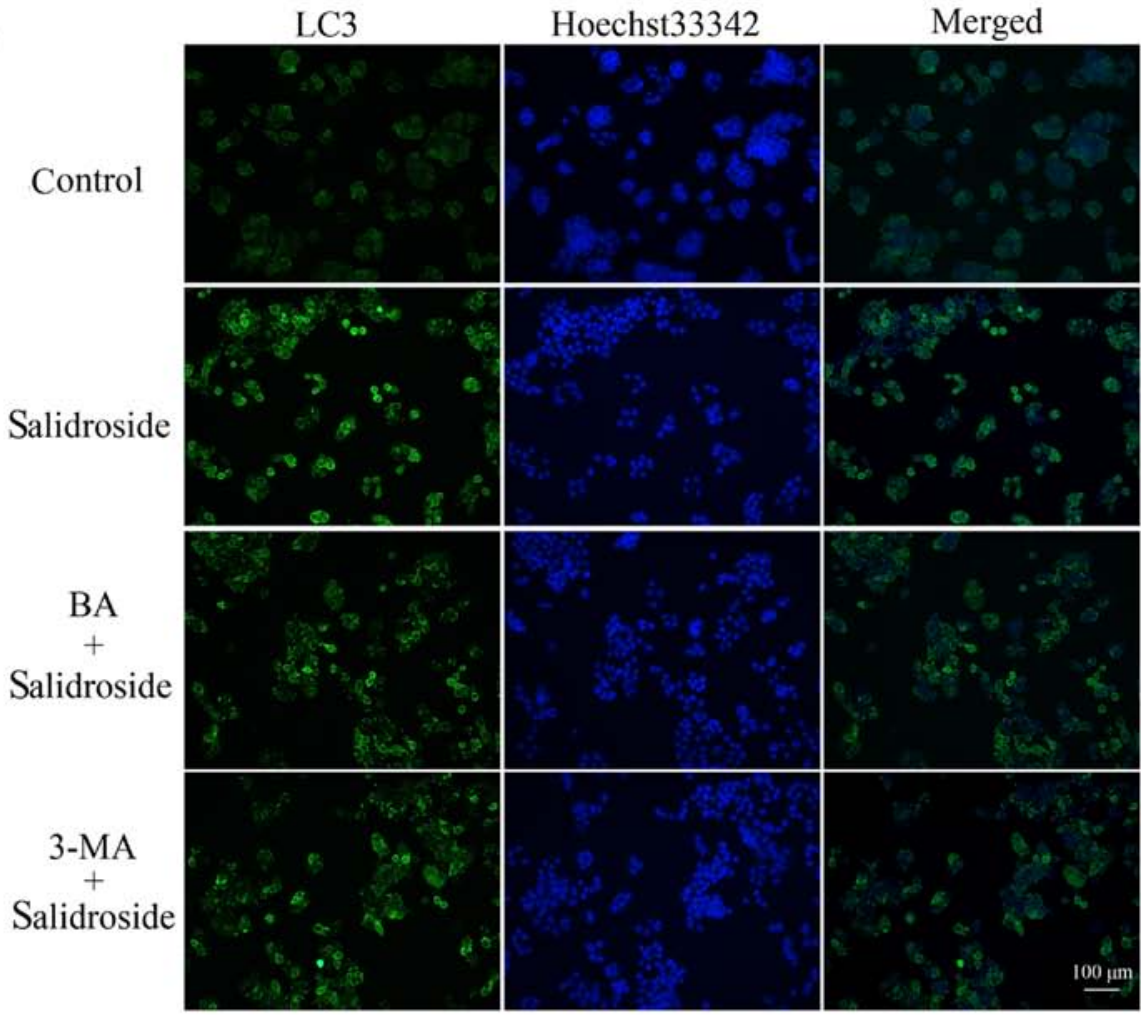

B
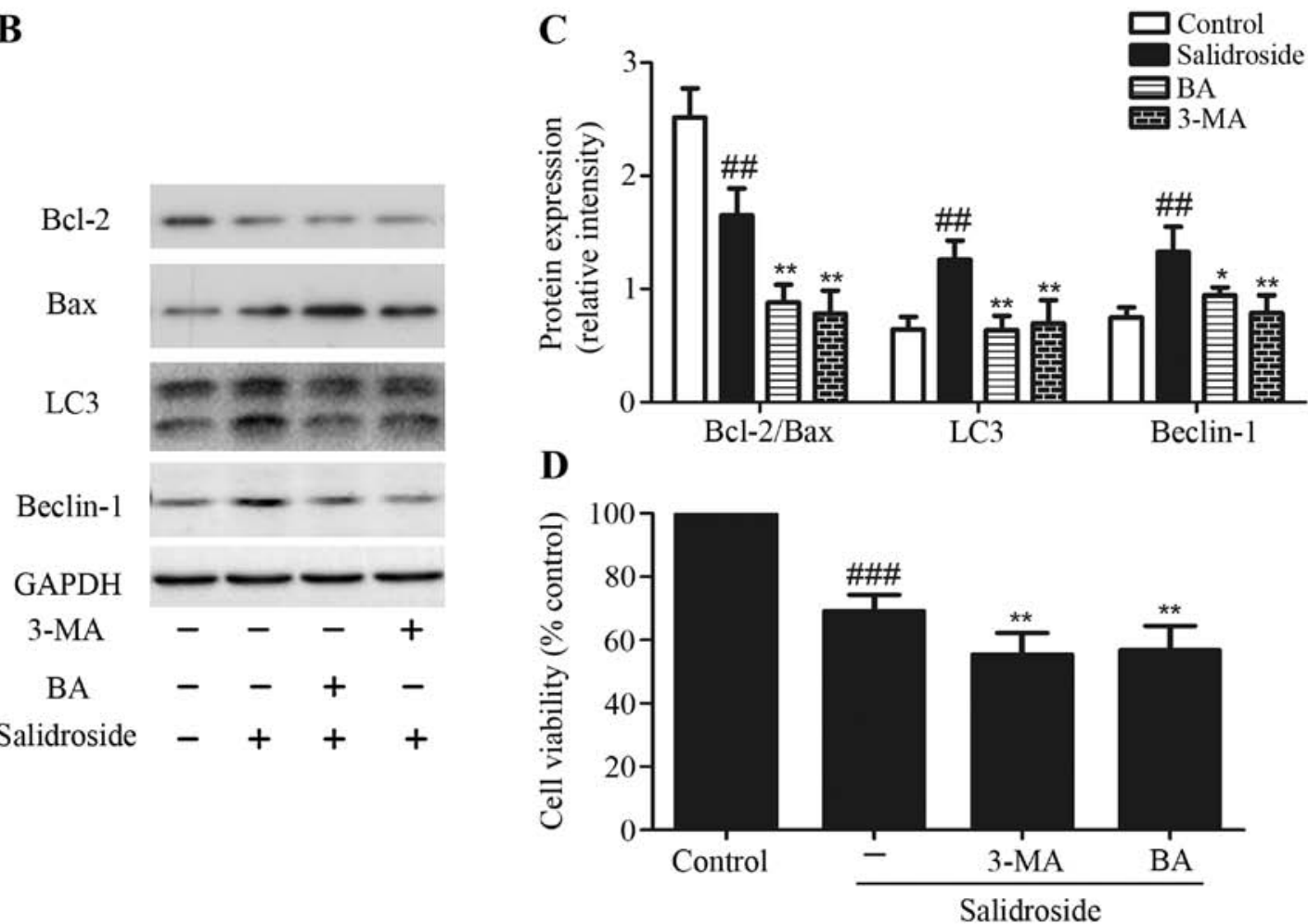

Figure 5. Inhibition of autophagy enhances apoptosis in salidroside-treated HT29 cells. (A) To confirm the autophagy induction by salidroside, cells were treated with $2 \mathrm{mM}$ salidroside for $48 \mathrm{~h}$ with or without pretreatment with $10 \mathrm{mM}$ 3-MA or $10 \mathrm{nM}$ BA for 30 min. Immunofluorescent staining was used to determine the $\mathrm{LC}^{+}$autophagic vacuole formation and results showed that 3-MA and BA prevented salidroside-induced autophagic vacuole formation. (B) Cells were treated as above before lysis. Western blot analyses were performed for checking the protein levels of Bcl-2, Bax, LC3 and Beclin-1. AntiGAPDH antibody was used for normalization. (C) Band intensity was quantified by densitometric analysis. (D) Cells were treated as above, cell viability was determined using MTT assay. Values indicate the mean $\pm \mathrm{SD}$. ${ }^{\# \#} \mathrm{p}<0.01,{ }^{\# \# \#} \mathrm{p}<0.001$ vs. control group; ${ }^{*} \mathrm{p}<0.05,{ }^{* *} \mathrm{p}<0.01$ vs. treated cells with salidroside only.

and $36.2 \%$ decline in the ratio of p-PI3K over PI3K, 19.6, 21.3 and $31.2 \%$ decrease in the ratio of p-Akt over Akt, and 30.5, 34.7 and $46.3 \%$ reduction in the ratio of p-mTOR over mTOR in HT29 colon cancer cells when treated with salidroside at $0.5,1$ and $2 \mathrm{mM}$, respectively. In addition, treatment of HT29 cells with $10 \mu \mathrm{M}$ LY294002 plus $2 \mathrm{mM}$ salidroside decreased 
A

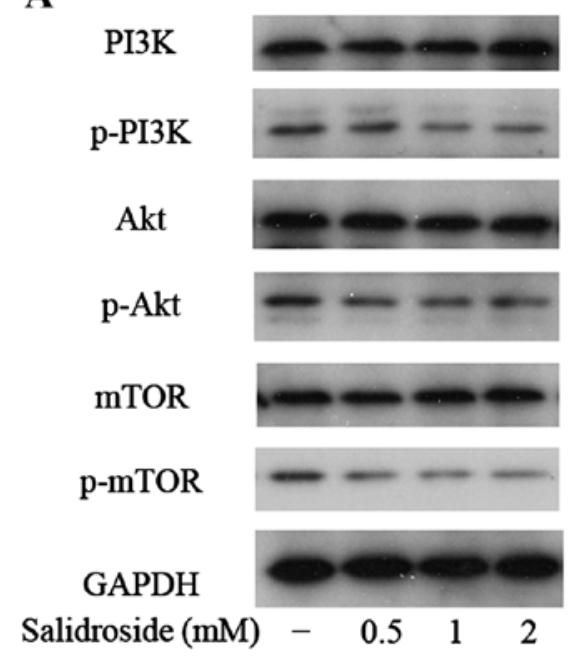

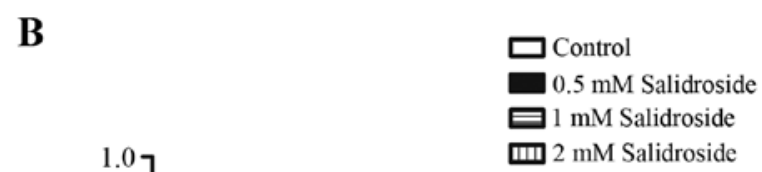

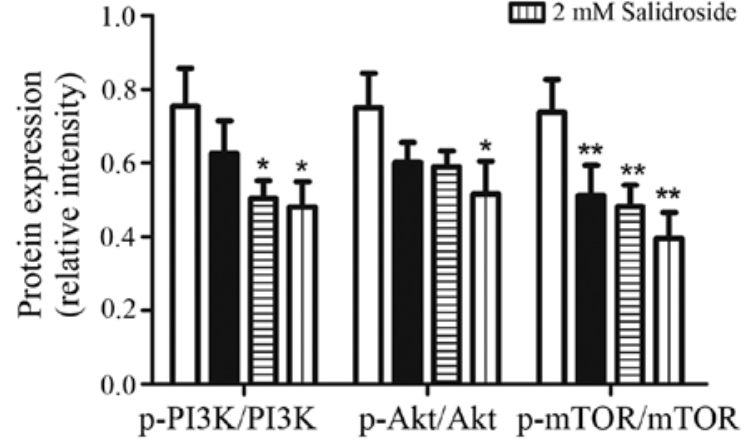

C

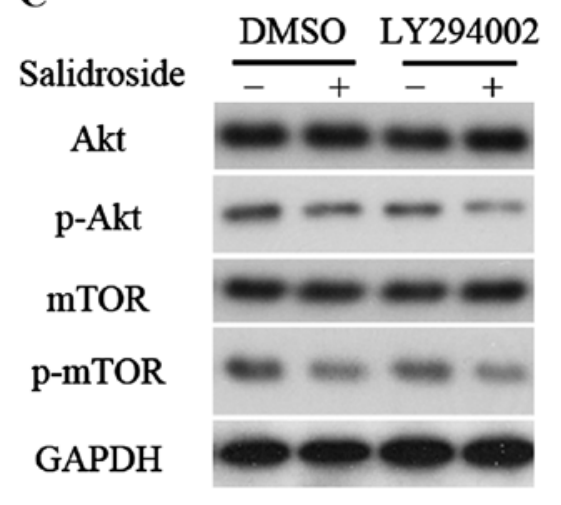

D

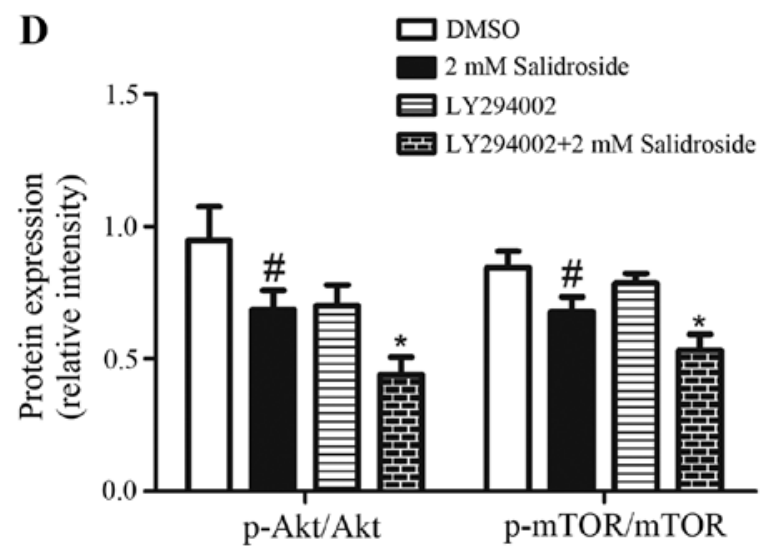

Figure 6. Salidroside inhibits the activation of PI3K/Akt/mTOR signaling pathway. (A) Representative blots showing the expression levels of p-PI3K, PI3K, p-Akt, Akt, p-mTOR and mTOR in HT29 colon cancer cells treated with 0.5, 1 and 2 mM salidroside for 48 h. Anti-GAPDH antibody was used for normalization. (B) The intensity of bands was quantified by densitometric analysis. All values represent mean \pm SD of three independent experiments. ${ }^{*}<<0.05$, ${ }^{* *}$ p $<0.01$ vs. control. (C) Representative blots showing the expression levels of p-Akt, Akt, p-mTOR and mTOR in HT29 colon cancer cells treated with $10 \mu \mathrm{M}$ LY294002 plus $2 \mathrm{mM}$ salidroside for $48 \mathrm{~h}$. Anti-GAPDH antibody was used for normalization. (D) The intensity of bands was quantified by densitometric analysis. All values represent mean \pm SD of three independent experiments. ${ }^{\#} \mathrm{p}<0.05$ vs. control, ${ }^{*}$ p $<0.05$ vs. salidroside group.

the ratio of $\mathrm{p}$-Akt/Akt and $\mathrm{p}$-mTOR/mTOR more than treatment with salidroside alone (Fig. 6C and D). Thus, these data suggested that the salidroside inhibited the activation of PI3K/ Akt/mTOR signaling pathway.

\section{Discussion}

In our research, we found that salidroside inhibited the growth of HT-29 human colorectal cancer cells concentration- and time-dependently, which is consistent with the results that salidroside inhibited proliferation, decreased the migration and invasion of SW1116 cells (25). However, whether the anticancer effect of salidroside is related with apoptosis and autophagy have not been elucidated.

Apoptosis is an active process in which apoptotic cells undergo chromatin condensation and fragmentation followed by the formation of apoptotic bodies (33). Several genes have been shown to regulate apoptosis. The proteins of the $\mathrm{Bcl}-2$ family represent a critical checkpoint in major apoptotic signal transduction cascades (34). In addition, apoptotic cell death is typically determined by the ratio of $\mathrm{Bcl}-2 / \mathrm{Bax}$ (35-37). In this study, we found that salidroside induced cell apoptosis, accompanied by an increase of chromatin condensation and nuclear fragmentation, and a decrease of Bcl-2/Bax expression ratio. We thus examined whether salidroside induced autophagy.

Autophagy in cancer has begun to be investigated and has been suggested as a novel potential target for improved anticancer therapies (38-40). Autophagy is characterized by engulfment of cytoplasm and organelles into doublemembrane bound structures, autophagosomes, and delivery to and subsequent degradation in lysosomes (41). Autophagy allows degradation of the cytoplasmic contents under certain stress conditions such as oxidative stress, nutrient starvation, misfolded protein accumulation, and irradiation is a temporary survival mechanism. Recent studies shown that autophagy is needed for cancer survival and tumorigenesis $(42,43)$. Autophagy can serve as a mechanism of self-defense by recycling essential molecules, and by contributing to therapy resistance (44-46). Conversely, autophagy can inhibit tumor progression (45). Extensive autophagy can also result in destruction of vital cell constituents, committing the cell to death (47). LC3 and ATG12, two ubiquitin-like protein 
systems, play an important role for the autophagosomal membrane formation and expansion (48). LC3 consists of two forms, LC3-I and its cleavage form, LC3-II. The conversion of soluble LC3-I to the membrane bound LC3 II is considered as one of the makers of autophagy induction in the cells. Detecting LC3-II by immunoblotting or immunofluorescence is a reliable method for monitoring autophagosome formation $(30,48,49)$. Beclin-1 has been well demonstrated to initiate autophagosome formation during autophagy (50). Autophagy is characterized morphologically by the formation of $\mathrm{LC}^{+}$ autophagic vacuoles and accumulation acidic vesicular organelles $(30,51)$. Our data showed that salidroside treatment increased the formation of $\mathrm{LC}^{+}$autophagic vacuoles and the accumulation of acidic vesicular organelles. Western blot analysis found that salidroside remarkably increased the ratio of LC3-II/LC3-I and Beclin-1 protein expression in a dosedependent manner. It is obviously that salidroside induced autophagy in HT29 colon cancer cells.

3-MA is a popular inhibitor of the autophagic agent. It has been reported to inhibit the activity of PI3-kinase (a kinase that is essential for vesicle nucleation, the first phase of autophagosome formation) and blocks the formation of preautophagosome, autophagosome, and autophagic vacuoles (52). BA, a known inhibitor of the late phase of autophagy, prevents maturation of autophagic vacuoles by inhibiting fusion between autophagosomes and lysosomes (53). Addition of 3-MA or BA attenuated the formation of $\mathrm{LC}^{+}$autophagic vacuoles and inhibited the increase of LC3-II/-I ratio and Beclin-1 protein expression induced by salidroside in HT29 colon cancer cells. Moreover, pre-treatment with 3-MA or BA augmented the inhibitory effects of salidoside on the expression of Bcl-2/Bax ratio and the cell viability. Collectively, these results indicated that inhibition of autophagy decreased cell viability and increased apoptosis, which revealed that autophagy provided a protective mechanism against salidroside-induced apoptosis.

Increasing evidence suggests that cross-talk between apoptosis and autophagy is made especially complicated by the fact that they share many common regulatory molecules, such as Bcl-2 and the PI3K/Akt/mTOR signaling pathway $(33,54)$. $\mathrm{PI} 3 \mathrm{~K}$ activates the downstream serine/threonine kinase Akt, which in turn, through a cascade of regulators, triggers the phosphorylation and activation of the serine/threonine kinase mTOR (55). PI3K/Akt/mTOR, a major intracellular signaling pathway, has received much attention in recent years given its potential role in cancer $(8,56)$. As a convergence point for a multitude of upstream signals, this critical pathway stimulates the activity of numerous downstream effectors and mediates enhanced cellular survival, growth, protein synthesis, motility, and other functions of pro-tumorigenic impact (57). Inhibition of PI3K/Akt/mTOR signaling pathway causes cell death associated with apoptosis and/or autophagy $(58,59)$. As shown by our western blot analysis, salidroside significantly decreased the activation of PI3K, Akt, and mTOR in HT-29 human colorectal cancer cells. In addition, PI3K inhibitor LY294002 could further inhibit the activation of Akt and mTOR induced by salidroside treatment. Thus, the inhibition of the PI3K/Akt/ mTOR signaling pathway contributes, at least in part, to the apoptosis-inducing and autophagy-inducing effect of salidroside in HT-29 human colorectal cancer cells.
In this study, we demonstrated the cell growth inhibitory effect of salidroside on HT-29 human colorectal cancer cells. We elucidated the underlying mechanism that involves cross-talk between apoptosis and autophagy via inhibition of PI3K/Akt/ mTOR signaling pathways. In conclusion, we have provided a basis for molecular mechanism of salidroside in colon cancer treatment. The potential application of salidroside in inhibiting colon cancer cell proliferation makes it an attractive agent for colorectal cancer research, and possibly treatment.

\section{Acknowledgements}

This study was supported by the Technological Innovation and Demonstration of Social Undertakings Projects (HS2014049) of Nantong, Jiangsu, China.

\section{References}

1. Torre LA, Bray F, Siegel RL, Ferlay J, Lortet-Tieulent J and Jemal A: Global cancer statistics, 2012. CA Cancer J Clin 65: 87-108, 2015.

2. Chaabane W, User SD, El-Gazzah M, Jaksik R, Sajjadi E, Rzeszowska-Wolny J and Los MJ: Autophagy, apoptosis, mitoptosis and necrosis: Interdependence between those pathways and effects on cancer. Arch Immunol Ther Exp (Warsz) 61: 43-58, 2013.

3. Su M, Mei Y and Sinha S: Role of the crosstalk between autophagy and apoptosis in cancer. J Oncol 2013: 102735-102748, 2013.

4. Woodle ES and Kulkarni S: Programmed cell death. Transplantation 66: 681-691, 1998.

5. Rubinsztein DC, Gestwicki JE, Murphy LO and Klionsky DJ: Potential therapeutic applications of autophagy. Nat Rev Drug Discov 6: 304-312, 2007.

6. Periyasamy-Thandavan S, Jiang M, Schoenlein P and Dong Z: Autophagy: Molecular machinery, regulation, and implications for renal pathophysiology. Am J Physiol Renal Physiol 297: F244-F256, 2009.

7. He $\mathrm{C}$ and Klionsky DJ: Regulation mechanisms and signaling pathways of autophagy. Annu Rev Genet 43: 67-93, 2009.

8. Li JP, Yang YX, Liu QL, Pan ST, He ZX, Zhang X, Yang T, Chen XW, Wang D, Qiu JX, et al: The investigational Aurora kinase A inhibitor alisertib (MLN8237) induces cell cycle G2/M arrest, apoptosis, and autophagy via p38 MAPK and Akt/mTOR signaling pathways in human breast cancer cells. Drug Des Devel Ther 9: 1627-1652, 2015.

9. Pan ST, Qin Y, Zhou ZW, He ZX, Zhang X, Yang T, Yang YX, Wang D, Qiu JX and Zhou SF: Plumbagin induces G2/M arrest, apoptosis, and autophagy via p38 MAPK- and PI3K/Akt/mTORmediated pathways in human tongue squamous cell carcinoma cells. Drug Des Devel Ther 9: 1601-1626, 2015.

10. Liu YL, Yang PM, Shun CT, Wu MS, Weng JR and Chen CC: Autophagy potentiates the anti-cancer effects of the histone deacetylase inhibitors in hepatocellular carcinoma. Autophagy 6: 1057-1065, 2010.

11. Jiang Q, Li F, Shi K, Yang Y and Xu C: Sodium selenite-induced activation of DAPK promotes autophagy in human leukemia HL60 cells. BMB Rep 45: 194-199, 2012.

12. Zhang X, Chen LX, Ouyang L, Cheng Y and Liu B: Plant natural compounds: Targeting pathways of autophagy as anti-cancer therapeutic agents. Cell Prolif 45: 466-476, 2012.

13. Burada F, Nicoli ER, Ciurea ME, Uscatu DC, Ioana M and Gheonea DI: Autophagy in colorectal cancer: An important switch from physiology to pathology. World J Gastrointest Oncol 7: 271-284, 2015.

14. Panossian A, Wikman G and Sarris J: Rosenroot (Rhodiola rosea): Traditional use, chemical composition, pharmacology and clinical efficacy. Phytomedicine 17: 481-493, 2010.

15. Hung SK, Perry R and Ernst E: The effectiveness and efficacy of Rhodiola rosea $\mathrm{L}$.: A systematic review of randomized clinical trials. Phytomedicine 18: 235-244, 2011.

16. Liu Z, Li X, Simoneau AR, Jafari M and Zi X: Rhodiola rosea extracts and salidroside decrease the growth of bladder cancer cell lines via inhibition of the mTOR pathway and induction of autophagy. Mol Carcinog 51: 257-267, 2012. 
17. Tu Y, Roberts L, Shetty K and Schneider SS: Rhodiola crenulata induces death and inhibits growth of breast cancer cell lines. J Med Food 11: 413-423, 2008.

18. Hu X, Lin S, Yu D, Qiu S, Zhang X and Mei R: A preliminary study: The anti-proliferation effect of salidroside on different human cancer cell lines. Cell Biol Toxicol 26: 499-507, 2010.

19. Hu X, Zhang X, Qiu S, Yu D and Lin S: Salidroside induces cellcycle arrest and apoptosis in human breast cancer cells. Biochem Biophys Res Commun 398: 62-67, 2010.

20. Liu X, Peng X, Hu Z, Zhao Q, He J, Li J and Zhong X: Effects of over-expression of ANXA10 gene on proliferation and apoptosis of hepatocellular carcinoma cell line HepG2. J Huazhong Univ Sci Technolog Med Sci 32: 669-674, 2012.

21. Wang J, Li JZ, Lu AX, Zhang KF and Li BJ: Anticancer effect of salidroside on A549 lung cancer cells through inhibition of oxidative stress and phospho-p38 expression. Oncol Lett 7: 1159-1164, 2014.

22. Skopińska-Rózewska E, Malinowski M, Wasiutyński A, Sommer E, Furmanowa M, Mazurkiewicz M and Siwicki AK: The influence of Rhodiola quadrifida $50 \%$ hydro-alcoholic extract and salidroside on tumor-induced angiogenesis in mice. Pol J Vet Sci 11: 97-104, 2008.

23. Sun C, Wang Z, Zheng Q and Zhang H: Salidroside inhibits migration and invasion of human fibrosarcoma HT1080 cells Phytomedicine 19: 355-363, 2012.

24. Zhang Y, Yao Y, Wang H, Guo Y, Zhang H and Chen L: Effects of salidroside on glioma formation and growth inhibition together with improvement of tumor microenvironment. Chin J Cancer Res 25: 520-526, 2013.

25. Sun KX, Xia HW and Xia RL: Anticancer effect of salidroside on colon cancer through inhibiting JAK2/STAT3 signaling pathway. Int J Clin Exp Pathol 8: 615-621, 2015.

26. Wang XW and Zhang YJ: Targeting mTOR network in colorectal cancer therapy. World J Gastroenterol 20: 4178-4188, 2014.

27. Morgensztern D and McLeod HL: PI3K/Akt/mTOR pathway as a target for cancer therapy. Anticancer Drugs 16: 797-803, 2005.

28. Maira SM, Furet P and Stauffer F: Discovery of novel anticancer therapeutics targeting the PI3K/Akt/mTOR pathway. Future Med Chem 1: 137-155, 2009

29. $\mathrm{Wu} \mathrm{P}$ and $\mathrm{Hu} \mathrm{YZ}$ : PI3K/Akt/mTOR pathway inhibitors in cancer: A perspective on clinical progress. Curr Med Chem 17: 4326-4341, 2010

30. Kabeya Y, Mizushima N, Ueno T, Yamamoto A, Kirisako T, Noda T, Kominami E, Ohsumi Y and Yoshimori T: LC3, a mammalian homologue of yeast Apg8p, is localized in autophagosome membranes after processing. EMBO J 19: 5720-5728, 2000.

31. Tanida I, Minematsu-Ikeguchi N, Ueno T and Kominami E: Lysosomal turnover, but not a cellular level, of endogenous LC3 is a marker for autophagy. Autophagy 1: 84-91, 2005.

32. Zhou ZW, Li XX, He ZX, Pan ST, Yang Y, Zhang X, Chow K Yang T, Qiu JX, Zhou Q, et al: Induction of apoptosis and autophagy via sirtuin1- and PI3K/Akt/mTOR-mediated pathways by plumbagin in human prostate cancer cells. Drug Des Devel Ther 9: 1511-1554, 2015.

33. Tsai JP, Lee CH, Ying TH, Lin CL, Lin CL, Hsueh JT and Hsieh YH: Licochalcone A induces autophagy through PI3K/ Akt/mTOR inactivation and autophagy suppression enhances Licochalcone A-induced apoptosis of human cervical cancer cells. Oncotarget 6: 28851-28866, 2015

34. Adams JM and Cory S: The Bcl-2 protein family: Arbiters of cell survival. Science 281: 1322-1326, 1998.

35. Korsmeyer SJ: Regulators of cell death. Trends Genet 11: 101-105, 1995.

36. Yang E and Korsmeyer SJ: Molecular thanatopsis: A discourse on the BCL2 family and cell death. Blood 88: 386-401, 1996.

37. Bar-Am O, Weinreb O, Amit T and Youdim MB: Regulation of Bcl-2 family proteins, neurotrophic factors, and APP processing in the neurorescue activity of propargylamine. FASEB J 19 1899-1901, 2005.

38. Hsuan SW, Chyau CC, Hung HY, Chen JH and Chou FP: The induction of apoptosis and autophagy by Wasabia japonica extract in colon cancer. Eur J Nutr 55: 491-503, 2016.

39. Xie CM, Chan WY, Yu S, Zhao J and Cheng CH: Bufalin induces autophagy-mediated cell death in human colon cancer cells through reactive oxygen species generation and JNK activation. Free Radic Biol Med 51: 1365-1375, 2011.
40. Yim NH, Jung YP, Kim A, Ma CJ, Cho WK and Ma JY: Oyaksungisan, a traditional herbal formula, inhibits cell proliferation by induction of autophagy via JNK activation in human colon cancer cells. Evid Based Complement Alternat Med 2013 231874-231883, 2013.

41. Mizushima N, Levine B, Cuervo AM and Klionsky DJ: Autophagy fights disease through cellular self-digestion. Nature 451: 1069-1075, 2008

42. Yang S, Wang X, Contino G, Liesa M, Sahin E, Ying H, Bause A, Li Y, Stommel JM, Dell'antonio G, et al: Pancreatic cancers require autophagy for tumor growth. Genes Dev 25: 717-729, 2011

43. Guo JY, Chen HY, Mathew R, Fan J, Strohecker AM, KarsliUzunbas G, Kamphorst JJ, Chen G, Lemons JM, Karantza V, et al: Activated Ras requires autophagy to maintain oxidative metabolism and tumorigenesis. Genes Dev 25: 460-470, 2011.

44. Ellington AA, Berhow MA and Singletary KW: Inhibition of Akt signaling and enhanced ERK1/2 activity are involved in induction of macroautophagy by triterpenoid B-group soyasaponins in colon cancer cells. Carcinogenesis 27: 298-306, 2006.

45. Gozuacik D and Kimchi A: Autophagy as a cell death and tumor suppressor mechanism. Oncogene 23: 2891-2906, 2004.

46. Ogier-Denis E and Codogno P: Autophagy: A barrier or an adaptive response to cancer. Biochim Biophys Acta 1603: 113-128, 2003

47. Clarke PG: Developmental cell death: Morphological diversity and multiple mechanisms. Anat Embryol (Berl) 181: 195-213, 1990.

48. Kaser A and Blumberg RS: Autophagy, microbial sensing, endoplasmic reticulum stress, and epithelial function in inflammatory bowel disease. Gastroenterology 140: 1738-1747, 2011.

49. Klionsky DJ, Abdalla FC, Abeliovich H, Abraham RT, AcevedoArozena A, Adeli K, Agholme L, Agnello M, Agostinis P, Aguirre-Ghiso JA, et al: Guidelines for the use and interpretation of assays for monitoring autophagy. Autophagy 8: 445-544, 2012.

50. Cao Y and Klionsky DJ: Physiological functions of Atg6/ Beclin 1: A unique autophagy-related protein. Cell Res 17: 839-849, 2007

51. Paglin S, Hollister T, Delohery T, Hackett N, McMahill M, Sphicas E, Domingo D and Yahalom J: A novel response of cancer cells to radiation involves autophagy and formation of acidic vesicles. Cancer Res 61: 439-444, 2001.

52. Petiot A, Ogier-Denis E, Blommaart EF, Meijer AJ and Codogno P: Distinct classes of phosphatidylinositol 3'-kinases are involved in signaling pathways that control macroautophagy in HT-29 cells. J Biol Chem 275: 992-998, 2000.

53. Yamamoto A, Tagawa Y, Yoshimori T, Moriyama Y, Masaki R and Tashiro Y: Bafilomycin A1 prevents maturation of autophagic vacuoles by inhibiting fusion between autophagosomes and lysosomes in rat hepatoma cell line, H-4-II-E cells. Cell Struct Funct 23: 33-42, 1998

54. Zhang DM, Liu JS, Deng LJ, Chen MF, Yiu A, Cao HH, Tian HY, Fung KP, Kurihara H, Pan JX, et al: Arenobufagin, a natural bufadienolide from toad venom, induces apoptosis and autophagy in human hepatocellular carcinoma cells through inhibition of PI3K/Akt/mTOR pathway. Carcinogenesis 34: 1331-1342, 2013.

55. Rodon J, Dienstmann R, Serra V and Tabernero J: Development of PI3K inhibitors: Lessons learned from early clinical trials. Nat Rev Clin Oncol 10: 143-153,2013.

56. Wang F, Wang Q, Zhou ZW, Yu SN, Pan ST, He ZX, Zhang X, Wang D, Yang YX, Yang T, et al: Plumbagin induces cell cycle arrest and autophagy and suppresses epithelial to mesenchymal transition involving PI3K/Akt/mTOR-mediated pathway in human pancreatic cancer cells. Drug Des Devel Ther 9: 537-560, 2015.

57. Vivanco I and Sawyers CL: The phosphatidylinositol 3-kinase AKT pathway in human cancer. Nat Rev Cancer 2: 489-501, 2002.

58. Wang K, Liu R, Li J, Mao J, Lei Y, Wu J, Zeng J, Zhang T, Wu H, Chen L, et al: Quercetin induces protective autophagy in gastric cancer cells: Involvement of Akt-mTOR- and hypoxia-induced factor 1 $\alpha$-mediated signaling. Autophagy 7: 966-978, 2011.

59. Shrivastava A, Kuzontkoski PM, Groopman JE and Prasad A: Cannabidiol induces programmed cell death in breast cancer cells by coordinating the cross-talk between apoptosis and autophagy. Mol Cancer Ther 10: 1161-1172, 2011. 